\title{
A COUNTEREXAMPLE TO MATSUMOTO'S CONJECTURE REGARDING ABSOLUTE LENGTH VS. RELATIVE LENGTH IN FINSLER MANIFOLDS
}

\author{
JEANNE N. CLELLAND
}

\begin{abstract}
Matsumoto conjectured that for any Finsler manifold $(M, F)$ for which the restriction of the fundamental tensor to the indicatrix of $F$ is positive definite, the absolute length $F(X)$ of any tangent vector $X \in T_{x} M$ is the global minimum for the relative length $|X|_{y}$ as $y$ varies along the indicatrix $I_{x} \subset T_{x} M$ of $F$. In this note, we disprove this conjecture by presenting a counterexample.
\end{abstract}

\section{Preliminaries}

Let $M$ be a smooth manifold of dimension $n$. Let $x=\left(x^{1}, \ldots, x^{n}\right)$ be a local coordinate system on $M$, and let $(x, y)=\left(x^{1}, \ldots, x^{n}, y^{1}, \ldots, y^{n}\right)$ be the corresponding canonical local coordinate system on the tangent bundle TM. A Finsler metric on $M$ is a function

$$
F: T M \rightarrow[0, \infty)
$$

with the following properties:

(1) Regularity: $F$ is $C^{\infty}$ on the slit tangent bundle $T M \backslash\{0\}$.

(2) Positive homogeneity: $F(x, \lambda y)=\lambda F(x, y)$ for all $\lambda>0$.

(3) Strong convexity: The fundamental tensor $g=\left[g_{i j}\right]$, defined by

$$
g_{i j}(x, y)=\frac{1}{2} \frac{\partial^{2}\left(F^{2}\right)}{\partial y^{i} \partial y^{j}}
$$

is positive definite at every point of $T M \backslash\{0\}$.

(For details, see [1.) In other words, a Finsler metric on a manifold $M$ is a smoothly varying Minkowski norm on each tangent space $T_{x} M$. A Finsler metric is Riemannian if and only if the fundamental tensor $g$ is independent of the $y$-coordinates, in which case we have

$$
F^{2}(x, y)=g_{i j}(x) y^{i} y^{j} .
$$

A Finsler manifold is a pair $(M, F)$ where $M$ is a smooth manifold and $F$ is a Finsler metric on $M$.

Definition 1. Let $(M, F)$ be a Finsler manifold.

- For any tangent vector $X \in T_{x} M$, the length of $X$, also known as the absolute length of $X$, is defined to be the value of $F(x, X)$.

Key words and phrases. Finsler manifold, absolute length, relative length, counterexample.

The author was partially supported by a Collaboration Grant for Mathematicians from The Simons Foundation. 
- For any $x \in M$, the indicatrix at $x$ is the subset $I_{x} \subset T_{x} M$ defined by

$$
I_{x}=\left\{y \in T_{x} M \mid F(x, y)=1\right\} .
$$

The indicatrix is the Finsler analog of the unit sphere in each tangent space.

In [2], Matsumoto introduced the following notion of relative length:

Definition 2. For any tangent vector $X \in T_{x} M$ and any vector $y \in I_{x}$, the relative length $|X|_{y}$ of $X$ with respect to $y$ is defined by

$$
|X|_{y}=\sqrt{g_{i j}(x, y) X^{i} X^{j}}
$$

It follows from the positive homogeneity property of Finsler metrics that for any $X \in I_{x}$, $|X|_{X}=F(X)=1$. More generally, for any nonzero $X \in T_{x} M$, if we set $\bar{X}=\frac{X}{F(X)}$, then $|X|_{\bar{X}}=F(X)$.

Based on this definition, Matsumoto defined the relative energy function of a given tangent vector $X \in T_{x} M$ to be the function $E_{X}: I_{x} \rightarrow \mathbb{R}$ defined by

$$
E_{X}(y)=\frac{1}{2}|X|_{y}^{2}
$$

Matsumoto explored the behavior of the critical points of the relative energy function and made the following conjecture:

Conjecture 1. For any nonzero $X \in T_{x} M$, the relative energy function $E_{X}: I_{x} \rightarrow \mathbb{R}$ achieves its global minimum at the point $y=\frac{X}{F(X)}$; equivalently, $F(X) \leq|X|_{y}$ for all $y \in I_{x}$.

This conjecture was stated somewhat informally, with the caveat "provided that [the metric] satisfy the usual desirable assumptions." It appears from the remainder of the paper [2] that this comment refers to the strong convexity condition for Finsler metrics, and that Matsumoto also considered more general metrics for which this condition does not necessarily hold everywhere.

\section{A COUnterexample}

In [2], Matsumoto proved Conjecture 1 for cubic Finsler metrics, i.e., metrics of the form

$$
F(x, y)=\sqrt[3]{a_{i j k}(x) y^{i} y^{j} y^{k}}
$$

under the additional assumption that the induced metric of the indicatrix is positive definite. (We note that this positivity condition is an immediate consequence of the strong convexity condition.) However, the indicatrix $I_{x}$ of a metric of this form - or of an analogous $m$ th-root metric for any odd $m$-in any tangent space $T_{x} M$ is never convex, or even compact, and so this positivity condition can never hold on all of $I_{x}$. This also means that, strictly speaking, such a metric can never be Finsler.

In this section, we present a counterexample to Conjecture 1. Let $M$ be a 2-dimensional Minkowski space with local coordinates $y=\left(y_{1}, y_{2}\right)$ (we use subscripts rather than superscripts in order to distinguish more easily between indices and exponents), and consider the 
4th-root metric

$$
F(y)=\sqrt[4]{y_{1}^{4}+3 y_{1}^{2} y_{2}^{2}+y_{2}^{4}}
$$

on $M$. The indicatrix of this metric is shown in Figure 1 .

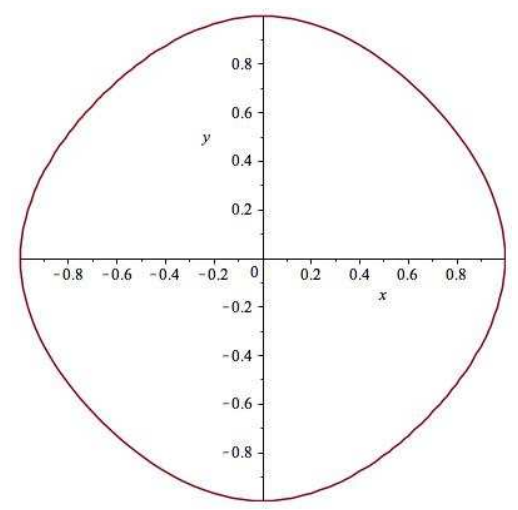

FiguRE 1. Indicatrix for the metric $F(y)=\sqrt[4]{y_{1}^{4}+3 y_{1}^{2} y_{2}^{2}+y_{2}^{4}}$

The fundamental tensor of $F$ is readily computed to be

$$
g=\frac{1}{2\left(y_{1}^{4}+3 y_{1}^{2} y_{2}^{2}+y_{2}^{4}\right)^{3 / 2}}\left[\begin{array}{cc}
2 y_{1}^{6}+9 y_{1}^{4} y_{2}^{2}+6 y_{1}^{2} y_{2}^{4}+3 y_{2}^{6} & 5 y_{1}^{3} y_{2}^{3} \\
5 y_{1}^{3} y_{2}^{3} & 3 y_{1}^{6}+6 y_{1}^{4} y_{2}^{2}+9 y_{1}^{2} y_{2}^{4}+2 y_{2}^{6}
\end{array}\right] .
$$

We have

$$
\operatorname{trace}(g)=\frac{5\left(y_{1}^{2}+y_{2}^{2}\right)^{3}}{2\left(y_{1}^{4}+3 y_{1}^{2} y_{2}^{2}+y_{2}^{4}\right)^{3 / 2}}, \quad \operatorname{det}(g)=\frac{3\left(2 y_{1}^{4}+y_{1}^{2} y_{2}^{2}+2 y_{2}^{4}\right)}{4\left(y_{1}^{4}+3 y_{1}^{2} y_{2}^{2}+y_{2}^{4}\right)},
$$

and since both of these expressions are strictly positive away from the origin, it follows that $g$ is positive definite at every point of $M \backslash\{0\}$. Therefore, its restriction to the indicatrix $I$ of $F$ is positive definite as well.

Now let $X=\left(X_{1}, X_{2}\right) \in M$, and consider the relative energy function

$$
\begin{aligned}
& E_{X}(y)=\frac{1}{2}\left(g_{11}(y) X_{1}^{2}+2 g_{12}(y) X_{1} X_{2}+g_{22}(y) X_{2}^{2}\right) \\
& =\frac{\left(2 X_{1}^{2}+3 X_{2}^{2}\right) y_{1}^{6}+\left(9 X_{1}^{2}+6 X_{2}^{2}\right) y_{1}^{4} y_{2}^{2}+10 X_{1} X_{2} y_{1}^{3} y_{2}^{3}+\left(6 X_{1}^{2}+9 X_{2}^{2}\right) y_{1}^{2} y_{2}^{4}+\left(3 X_{1}^{2}+2 X_{2}^{2}\right) y_{2}^{6}}{4\left(y_{1}^{4}+3 y_{1}^{2} y_{2}^{2}+y_{2}^{4}\right)^{3 / 2}} .
\end{aligned}
$$

Note that the denominator of $E_{X}$ is constant on the indicatrix $I$. Therefore, in order to find critical points of $E_{X}$ on $I$, it suffices to find critical points of the numerator (4)

$$
\begin{aligned}
& \tilde{E}_{X}(y)= \\
& \left(2 X_{1}^{2}+3 X_{2}^{2}\right) y_{1}^{6}+\left(9 X_{1}^{2}+6 X_{2}^{2}\right) y_{1}^{4} y_{2}^{2}+10 X_{1} X_{2} y_{1}^{3} y_{2}^{3}+\left(6 X_{1}^{2}+9 X_{2}^{2}\right) y_{1}^{2} y_{2}^{4}+\left(3 X_{1}^{2}+2 X_{2}^{2}\right) y_{2}^{6},
\end{aligned}
$$

subject to the constraint equation

$$
F^{4}(y)=y_{1}^{4}+3 y_{1}^{2} y_{2}^{2}+y_{2}^{4}=1
$$


that defines the indicatrix. To accomplish this, we use a Lagrange multiplier approach: We compute

$$
\begin{gathered}
\nabla \tilde{E}_{X}=\left[\begin{array}{c}
y_{1}\left(\left(12 X_{1}^{2}+18 X_{2}^{2}\right)\left(y_{1}^{4}+y_{2}^{4}\right)+\left(36 X_{1}^{2}+24 X_{2}^{2}\right) y_{1}^{2} y_{2}^{2}+30 X_{1} X_{2} y_{1} y_{2}^{3}\right) \\
y_{2}\left(\left(18 X_{1}^{2}+12 X_{2}^{2}\right)\left(y_{1}^{4}+y_{2}^{4}\right)+30 X_{1} X_{2} y_{1}^{3} y_{2}+\left(24 X_{1}^{2}+36 X_{2}^{2}\right) y_{1}^{2} y_{2}^{2}\right)
\end{array}\right], \\
\nabla\left(F^{4}\right)=\left[\begin{array}{l}
y_{1}\left(4 y_{1}^{2}+6 y_{2}^{2}\right) \\
y_{2}\left(6 y_{1}^{2}+4 y_{2}^{2}\right)
\end{array}\right] .
\end{gathered}
$$

Critical points occur wherever the vectors $\nabla \tilde{E}_{X}$ and $\nabla\left(F^{4}\right)$ are linearly dependent. A straightforward computation shows that this occurs precisely when

$$
y_{1} y_{2}\left(y_{1}-y_{2}\right)\left(y_{1}+y_{2}\right)\left(y_{1}^{2}+y_{2}^{2}\right)\left(X_{1} y_{2}-X_{2} y_{1}\right)^{2}=0 \text {. }
$$

Therefore, the critical points $\bar{y}=\left(\bar{y}_{1}, \bar{y}_{2}\right) \in I$ are those points where any of the following hold:

- $\bar{y}$ is a scalar multiple of $X$;

- $\bar{y}_{1}=0, \bar{y}_{2} \neq 0$

- $\bar{y}_{1} \neq 0, \bar{y}_{2}=0$;

- $\bar{y}_{1}= \pm \bar{y}_{2}$.

Evaluating the relative energy function (3) at these points yields the critical values

$$
\begin{aligned}
E_{X}(X) & =\sqrt{X_{1}^{4}+3 X_{1}^{2} X_{2}^{2}+X_{2}^{4}}=\frac{1}{2} F^{2}(X), \\
E_{X}\left(\bar{y}_{1}, 0\right) & =\frac{1}{2} X_{1}^{2}+\frac{3}{4} X_{2}^{2}, \\
E_{X}\left(0, \bar{y}_{2}\right) & =\frac{3}{4} X_{1}^{2}+\frac{1}{2} X_{2}^{2}, \\
E_{X}\left(\bar{y}_{1}, \bar{y}_{1}\right) & =\frac{1}{2 \sqrt{5}}\left(2 X_{1}^{2}+X_{1} X_{2}+X_{2}^{2}\right), \\
E_{X}\left(\bar{y}_{1},-\bar{y}_{1}\right) & =\frac{1}{2 \sqrt{5}}\left(2 X_{1}^{2}-X_{1} X_{2}+X_{2}^{2}\right) .
\end{aligned}
$$

(We note that, since the relative energy function (3) is homogeneous of degree zero in $y$, it is not necessary to scale the critical points so that $F(\bar{y})=1$ in order to compute the critical values.)

Example 1. Let $X=(1,0)$. Then from (7), we have

$$
E_{X}(X)=E_{X}\left(\bar{y}_{1}, 0\right)=\frac{1}{2}, \quad E_{X}\left(0, \bar{y}_{2}\right)=\frac{3}{4}, \quad E_{X}\left(\bar{y}_{1}, \pm \bar{y}_{1}\right)=\frac{1}{\sqrt{5}},
$$

so $E_{X}$ has neither a global maximum nor a global minimum at $X$.

The graph of $E_{X}$ as a function of the Euclidean angle parameter $\theta=\tan ^{-1}\left(\frac{y_{2}}{y_{1}}\right)$ along $I$ is shown in Figure 2, The scalar multiples of $X$ in $I$ correspond to $\theta \in\{0, \pi, 2 \pi\}$ and are indicated on the graph. 


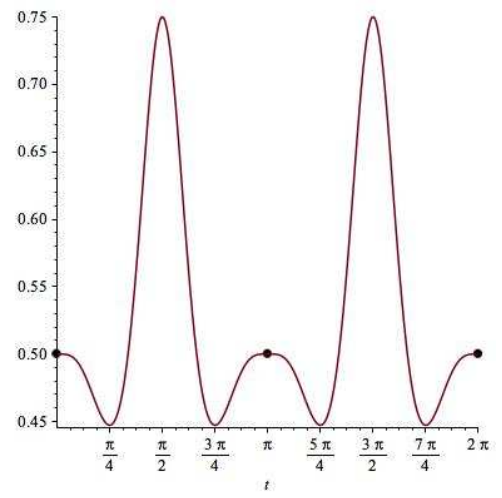

Figure 2. Graph of $E_{X}$ for $X=(1,0)$

Example 2. For an example where $X$ is not one of the "universal" critical points, let $X=(1,3)$. Then from (7), we have

$$
\begin{gathered}
E_{X}(X)=\frac{\sqrt{109}}{2} \approx 5.22, \quad E_{X}\left(\bar{y}_{1}, 0\right)=\frac{29}{4}=7.25, \quad E_{X}\left(0, \bar{y}_{2}\right)=\frac{21}{4}=5.25, \\
E_{X}\left(\bar{y}_{1}, \bar{y}_{1}\right)=\frac{23}{2 \sqrt{5}} \approx 5.14, \quad E_{X}\left(\bar{y}_{1}, \bar{y}_{1}\right)=\frac{1}{\sqrt{5}} \approx 3.80,
\end{gathered}
$$

so again, $E_{X}$ has neither a global maximum nor a global minimum at $X$.

The graph of $E_{X}$ as a function of the Euclidean angle parameter $\theta=\tan ^{-1}\left(\frac{y_{2}}{y_{1}}\right)$ along $I$ is shown in Figure 3. The scalar multiples of $X$ in $I$ correspond to $\theta \in\left\{\tan ^{-1}(3), \tan ^{-1}(3)+\pi\right\}$ and are indicated on the graph. In this case, the critical points at scalar multiples of $X$ are not even local extrema of $E_{X}$, but rather are inflection points.

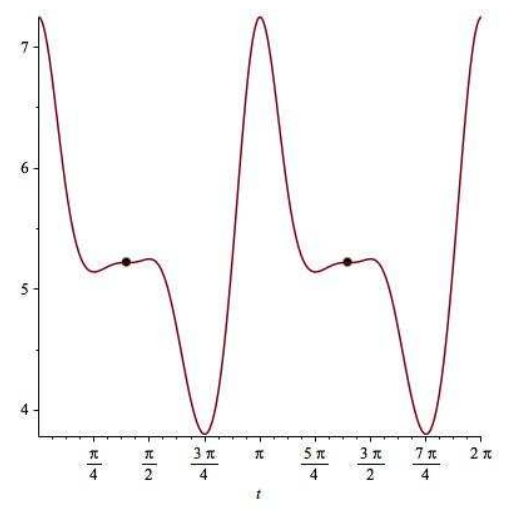

FiguRE 3. Graph of $E_{X}$ for $X=(1,3)$

These computations clearly demonstrate that Conjecture 1 does not hold for the metric (1), and hence is false in general. 


\section{REFERENCES}

[1] D. Bao, S.-S. Chern, and Z. Shen, An Introduction to Riemann-Finsler Geometry, Graduate Texts in Mathematics 200, Springer-Verlag, New York (2000).

[2] M. Matsumoto, "The length and the relative length of tangent vectors of Finsler spaces," Rep. Math. Phys. 15 (1979) 375-386.

Dept. of Mathematics, 395 UCB, University of Colorado, Boulder, CO 80309-0395, JEANNE.CLELLAND@COLORADO.EDU 